Meta

Journal des tradlucteurs

Translators' Journal

\title{
Réflexions sur la manière de traduire ou les trois états de la traduction
}

\section{Jean-Claude Gémar}

Volume 30, numéro 3, septembre 1985

URI : https://id.erudit.org/iderudit/002851ar

DOI : https://doi.org/10.7202/002851ar

Aller au sommaire du numéro

Éditeur(s)

Les Presses de l'Université de Montréal

ISSN

0026-0452 (imprimé)

1492-1421 (numérique)

Découvrir la revue

Citer cet article

Gémar, J.-C. (1985). Réflexions sur la manière de traduire ou les trois états de la traduction. Meta, 30(3), 236-241. https://doi.org/10.7202/002851ar d'utilisation que vous pouvez consulter en ligne.

https://apropos.erudit.org/fr/usagers/politique-dutilisation/ 


\title{
RÉFLEXIONS SUR LA MANIÈRE DE TRADUIRE OU LES LES TROIS ÉTATS DE LA TRADUCTION
}

\author{
JEAN-Claude GÉmaR
}

La place de la traduction dans l'histoire et le développement de la pensée est parfois sous-estimée, notamment lorsque l'on ne voit dans l'activité traduisante qu'un art mineur, un passe-temps de lettré. Or les fonctions de l'activité qui occupe depuis quelque 5000 ans $^{1}$ ceux qui se sont donnés pour tâche de faire connaître, en les "translatant " dans leur langue, les textes rédigés dans un idiome étranger, ne sont plus à démontrer, en particulier dans les domaines intellectuel, artistique et même linguisti$q^{2} e^{2}$. L'opération traduisante en revanche, bien qu'elle ait suscité de nombreux ouvrages et commentaires depuis un demi-siècle, et plus particulièrement depuis une trentaine d'années, semble avoir moins retenu l'attention des observateurs du langage, plus intéressés par la fonction de celui-ci que par la manière dont est rendu le message, dont la communication s'établit. Pourtant, les grands traducteurs ont tous d'une façon ou d'une autre réfléchi sur la manière de traduire, certains d'entre eux ont même exprimé sur leur art des idées originales et profondes. Aucun toutefois n'a exposé son propos de façon systématique ni cherché à tirer de son expérience les éléments constitutifs d'un système, les prémisses d'une philosophie de la traduction qui pussent servir de prolégomènes à une théorie de la manière de traduire, au sens moderne du mot.

Il faut attendre la fin du XIXe siècle et ses classifications, son souci de la hiérarchie linéaire et l'âge du positivisme pour voir s'amorcer une réflexion plus nourrie mais dont les racines, dans le trièdre épistémologique tracé par Foucault ${ }^{3}$, plongent encore aux sources de la réflexion philosophique traditionnelle plutôt qu'à celles des sciences empiriques ou même déductives qui, quelques décennies plus tard, allaient servir de fondement aux théories "scientifiques" les plus hardies. Le vide épistémologique caractérisant une des activités les plus nobles (et anciennes) de l'homme ne pouvait manquer d'attirer les théoriciens du langage dont les recherches sur les fonctions, le processus, le produit, la didactique, etc., de la traduction ont conféré à une pratique traditionnellement considérée comme un "art littéraire " plutôt qu'une "science " le statut, envié et redouté tout à la fois, de discipline quasi scientifique.

Cette évolution millénaire s'est faite au rythme des progrès de la science, des techniques et des sciences humaines et sociales en trois étapes principales dont la courbe, dans un premier temps, a suivi une progression linéaire, en gros des origines à la fin de la Première Guerre mondiale, qui s'est accélérée dans un deuxième temps sous l'impulsion de la pensée saussurienne avant de subir la foudroyante accélération que connaît l'activité traduisante depuis la Deuxième Guerre mondiale, et plus particulièrement depuis un quart de siècle. Au cours de cette évolution, la traduction est passée successivement par l'état théologique présaussurien, l'état métaphysique - linguistique - et l'état "scientifique" (ou plutôt : quasi scientifique) marqué par l'avènement de la machine à traduire et de l'intelligence artificielle. 


\section{L'ÉTAT THÉOLOGIQUE OU LE CARACTÈRE SACRÉ DU TEXTE}

Le traducteur est le premier et le seul artisan de son art comme en témoignent les très nombreux écrits que lui a inspirés l'opération traduisante ${ }^{4}$. $\mathrm{Si}$, dès les débuts, les traducteurs se scindent en deux groupes, deux écoles irréductiblement opposées quant à la manière de traduire, les uns prônant la traduction du signifié plutôt que du signifiant soit le mot à mot, soit le sens -, un choix fondamental s'esquisse déjà chez Cicéron à propos de la fonction didactique de la traduction : il faut alors " respecter leurs phrases [des auteurs grecs] avec les figures de mots ou de pensées... " 5

Ce respect de l'auteur, ce caractère sacré du texte, on les retrouve, amplifiés, chez un autre grand précurseur et traducteur, saint Jérôme. Dans son esquisse de traité de traduction, Lettre à Pammachius. De optime genere interpretandi, saint Jérôme défend avec ferveur sa conception de la manière de traduire "une idée par une idée " 6 mais fait une exception pour " les Saintes Écritures, où l'ordre des mots est aussi un mystère. "7 Cette conception particulière de la traduction littérale opposée à la traduction du sens est révélatrice de l'ambivalence de la nature humaine : le traducteur doit s'effacer devant le texte sacré, il ne saurait rivaliser avec le divin.

Ce caractère sacré du texte (et de son auteur) n'est pas particulier à une époque ni à une catégorie spécifique de traducteurs - par exemple, ceux qui traduisent des textes religieux - puisqu'on la retrouve tout au long de cette longue période de quelque 2000 ans qui va de Cicéron à Nabokov en passant entre autres par Chateaubriand - traducteur de Milton -, Baudelaire et Leconte de Lisle qui ont surtout traduit des textes littéraires. En ce sens il est possible de parler du caractère théologique de la traduction qui reflète le respect, voire la crainte qu'éprouve le traducteur devant l'œuvre qu'il entreprend de faire connaître. Comme l'a bien fait voir Georges Mounin dans les Belles infidèles :

Il s'agit premièrement de restituer, grâce à la littéralité la plus scrupuleuse, les façons de penser, de sentir, de parler, d'agir, de vivre et de chanter, des Grecs authentiques d'il y a trois mille ans. Cet espace entre eux et nous, que les « belles infidèles ", à l'époque classique, avaient presque supprimé, doit être maintenant rendu perceptible par tous les moyens.

Le littéralisme mystique, et même ethnographique par son objet, qui domine cette première période de l'histoire de la traduction refiète en partie la lente évolution d'un système en équilibre, quasiment statique depuis les origines, système dont le développement devait suivre les progrès de disciplines d'appoint et non les précéder. La traduction, moyen privilégié de transmission de la culture et de communication de la pensée durant des siècles, figure de proue des civilisations occidentales, est longtemps restée dans le sillage du vaisseau scientifique, dans l'antichambre de la société industrielle. Le $\mathrm{XIX}^{\mathrm{e}}$ siècle incarne tout particulièrement les contradictions où devait finir par s'enfermer le littéralisme érigé en "système de traduction ", en acte de foi parnassien : "l'art pour l'art ». La traduction littéraire voit diminuer son importance à mesure que se développent des domaines, autrefois inconnus, au même rythme que celui des sociétés engagées sur la voie du progrès scientifique et technique : le champ d'action du traducteur s'ouvre sur des horizons nouveaux et insoupçonnés. En somme, à l'ère préscientifique, la traduction était prête à accomplir sa première révolution industrielle qui devait la conduire de l'état théologique à celui de la métaphysique, autrement dit de la foi en un système fondé sur un positivisme que l'on voulait croire scientifique.

L'ÂGE DE LA MÉTAPHYSIQUE OU LE " POSITIVISME SCIENTIFIQUE "

Les anciens ont toujours pensé que la difficulté majeure de la traduction était d'ordre stylistique et non sémantique. La traduction littéraire, notamment poétique, illustre 
cette croyance. Les philosophes grecs croyaient que le sens pouvait être compris et exprimé. Cette illusion a longtemps servi d'inspiration aux traducteurs, en particulier aux tenants du littéralisme, pour les raisons évoquées plus haut. Le développement de la linguistique générale et interne qui a fait suite à la publication du Cours de linguistique générale (1916) de Ferdinand de Saussure marque une étape décisive dans l'histoire de la traduction. C'est à partir des orientations définies par ce grand linguiste que le monde de la traduction devait se scinder (provisoirement ?) en deux groupes antithétiques qui ont accentué le caractère manichéen du couple lettres/sciences, littéraire/scientifique (Anciens et Modernes ?) que le XVIIre siècle avait arbitrairement dissocié.

Cette deuxième période commence avec le $\mathrm{XX}^{\mathrm{e}}$ siècle, non celui, mathématique, de 1900, mais plutôt celui qui s'ouvre sur l'ère moderne que sanctionnent le Traité de Versailles et la fin de la "Belle Époque". Le vide épistémologique qui était alors le propre de l'activité traduisante ne pouvait rester indéfiniment béant. Il sera peu à peu comblé, en grande partie grâce aux efforts des théoriciens, linguistes pour la plupart : les « linguitrads ${ }^{8}$. En introduisant dans le débat théorique sur la composition du signe linguistique le couple SIGNIFIANT/SIGNIFIÉ, de Saussure jetait les bases de la linguistique moderne ainsi que les fondements d'une théorie générale de la traduction qui n'ont pas encore été vraiment ébranlés. Désormais, on commence à s'interroger avec de plus en plus d'insistance sur les problèmes de SENS que pose l'opération traduisante. Peu à peu, d'une simple "manière de traduire " on en arrive à dégager les prémisses d'un système, puis les problèmes théoriques à partir desquels vont se développer les principaux éléments d'une théorie générale de la traduction. En somme, la traduction, discipline littéraire au départ, semble s'acheminer vers le statut scientifique que ses partisans voudraient lui voir reconnaître. Cette remise en question du statut littéraire de l'activité du traducteur ne laisse pas d'étonner ce dernier tant les linguistes apportent du zèle à démonter les mécanismes de l'opération traduisante pour aboutir, en fin de compte, soit à l'impossibilité de traduire, soit à la possibilité — limitée — de faire passer d'une langue dans une autre l'essentiel du message enchâssé dans la langue de départ, en raison du bagage minimal d'universaux que partagent les principales langues véhiculaires du monde occidental.

Le fossé se creuse donc entre théoriciens et praticiens. Ces derniers, qui traduisent depuis toujours comprennent mal le "scandale " dénoncé par Georges Mounin, qui oppose les théoriciens entre eux. Ce scandale pourrait bien être, quant à moi, le fait que l'on entretienne le traducteur dans l'illusion que seule la théorie est en mesure de l'aider dans sa tâche et de lui faciliter le travail. On connaît la boutade de Vinay déclarant " qu'il n'y a pas eu de théoriciens dans notre discipline pendant toute la période qui s'étend de Babel à Babel $" 9$, soit jusque vers 1955. Il faut reconnaître qu'entre la théorie et la pratique, au début des années 50 , la théorie de la traduction en était encore au stade du plus léger que l'air quand le traducteur en était déjà, lui, à celui de l'avion à réaction! Il serait naif de croire cependant qu'aucune considération théorique n'est venue éclairer l'activité traduisante entre Ferdinand de Saussure, Fedorov, Vinay et Darbelnet et autres Nida ${ }^{10}$. Au contraire, de nombreux linguistes avaient montré la voie aux théoriciens en puissance de la traduction ${ }^{11}$. Néanmoins, force est d'admettre avec Mounin que la " traduction, comme phénomène et comme problème distinct de langage, est passée sous silence ${ }^{12}$. "Ce silence est rompu depuis qu'une avalanche d'ouvrages, linguistiques pour la plupart, est venue combler partiellement ce vide en quelques années, tant en Europe qu'en Amérique du Nord.

La nature "métaphysique " de ce nouveau positivisme scientifique (entendre : nul ne saurait bien traduire s'il ne s'appuie sur des principes " scientifiquement "établis) ressort clairement des limites des théories linguistiques de la traduction lorsque du stade 
théorique l'on passe à celui de l'application, pour ne rien dire de la vérification. Disonsle tout net : les "théories " de la traduction n'ont pas apporté au traducteur les règles, générales ou particulières, infaillibles qu'il peut appliquer systématiquement lorsque se présente dans l'opération de transfert une difficulté inattendue. Il faut alors reconnaître que « la linguistique qui revendique le statut de science exacte du langage n'a pas encore franchi le pas essentiel lui permettant de faire une unanimité sur ses présupposés propres ${ }^{13}$. " La science a besoin de certitude, de durée. Or une des caractéristiques de la traduction est sa nature fugitive : une traduction correspond à un moment précis de l'histoire linguistique d'un groupe et, en ce sens, la traduction est d'essence extemporanée. De là la difficulté de lui conférer un statut scientifique permanent, de dégager des normes, de formuler des principes généraux applicables à toutes les langues que l'on désire traduire. À cet égard on peút dire que la traduction, jusqu'au début des années 50, en grande partie à cause des théoriciens de la linguistique et prise au jeu du positivisme scientifique, était d'essence métaphysique. Ce n'est qu'à partir des travaux et des recherches qui ont marqué les vingt-cinq dernières années que l'on peut avancer que la traduction, à l'instar de disciplines comme la linguistique, la psychologie, la sociologie, la théorie de la communication, etc., est entrée dans l'ère scientifique.

\section{VERS L'ÉTAT SCIENTIFIQUE OU LA SCIENCE AU SERVICE DE LA TRADUCTION}

Une remarque de René Dubos illustre parfaitement l'évolution de la traduction au cours du dernier quart de siècle :

(Traduction)

Depuis deux cents ans, et plus particulièrement dans les vingt-cinq dernières années où l'accélération a été foudroyante, l'homme est intervenu dans l'ordre naturel avec une vigueur et une ampleur qui laissent présager l'avènement d'une ère nouvelle dans l'histoire de l'humanité /.../L'homme /.../s'est mis à remplacer l'incontrôlé par le contrôlé, le naturel par l'artificiel, le hasard par la certitude sur toute la planète. Et il le fait à une vitesse et avec une ampleur jamais vues dans l'histoire de l'humanité14.

Les théoriciens de la traduction lorsqu'ils eurent enfin compris que la linguistique interne ne pouvait à elle seule couvrir le champ de l'activité traduisante, se sont tournés vers d'autres disciplines, complémentaires ou d'appoint, pour tenter de démonter le mécanisme de l'opération traduisante et d'en expliquer le fonctionnement. L'homme ne communique pas seulement par la langue et la tentative de récupération de la traduction par la linguistique ayant avorté quand on eut compris que celle-ci " aide autant la traduction qu'elle la paralyse " 15 et que cette dernière fait intervenir d'autres disciplines aussi nombreuses que variées (philologie, ethnologie, psychologie, sociologie, psycholinguistique, théorie de la communication...), on s'aperçut que le système binaire saussurien, le couple royal signifiant/signifié - ou : structure de surface/structure(s) profonde(s)... - ne suffisait plus pour décrire la totalité de l'opération traduisante ni même pour dresser la carte ethnographique de la langue de départ. Un troisième intervenant dans le champ traductionnel classique emprunté à la linguistique (signifiant/signifié, langue/parole, performance/compétence) est soit le traducteur lui-même (cf. Kelly ${ }^{16}$ ), soit le "destinataire" de la traduction (cf. Nida ${ }^{17}$ ). En définitive, c'est le message qui est au carrefour des recherches actuelles et non plus uniquement la langue. $\mathrm{La}$ réalité extérieure aux considérations sur la langue est probablement aussi importante en traduction que peut l'être la linguistique (voir Seleskovitch, Pergnier, Ladmiral et Delisle ${ }^{18}$ ). Cette nouvelle réalité " ternaire " se fonde sur des bases tout aussi solides que celles des couples linguistiques classiques et répond peut-être mieux aux besoins spécifiques de la théorie générale de la traduction. Si Dryden avait reconnu trois manières de traduire, on s'accorde à voir la traduction comme résultat, opération ou comparaison et également 
selon le trièdre : Texte (auteur) - Traducteur (relais) — Lecteur (destinataire). Enfin, historiquement le traducteur partait de l'esprit du texte, était fidèle à la forme et tenait à respecter la vérité (voir saint Jérôme). Ces principes ternaires, par un étonnant retour du pendule, refont leur apparition.

Il est symptomatique de constater qu'un nombre croissant de théoriciens nourris de pratiques et de connaissances s'inspirent de disciplines extérieures à la linguistique ou paralinguistiques sont en train d'édifier un corps de doctrine traductionnelle où la linguistique tient un rôle toujours essentiel, certes, mais non plus exclusif, de science auxiliaire de la traduction, et non l'inverse. L'esprit scientifique qui sous-tend la recherche actuelle dans le domaine de la traduction s'inscrit dans une perspective objective qui contraste singulièrement avec celui qui présidait encore voici peu. Ce changement d'attitude constitue, à mon avis, un des événements de l'histoire de la théorie générale de la traduction. Avec la prudence et l'humilité qui caractérisent la recherche entreprise dans un domaine aussi délicat que peut l'être la traduction, les néo-théoriciens parlent des fondements, des théorèmes ou de l'analyse du phénomène que constitue l'opération traduisante. Le mot théorie est soigneusement évité, et c'est mieux ainsi car qui parle de théorie sous-entend : hypothèse vérifiée. Cette modestie est de rigueur dans une discipline dont les présupposés (notamment sémantiques) restent du domaine de la spéculation.

Si l'on ajoute à cela la percée, véritablement scientifique, elle, de l'intelligence artificielle et de l'informatique dans le domaine jusque-là réservé au traducteur et les virtualités qu'elle laisse supposer (soit ses multiples possibilités d'application ${ }^{19}$ ), on admettra que le traducteur fait œuvre d'apprenti sorcier et qu'il est, lui aussi, entré dans l'ère scientifique : son art, désormais hybride, n'est pas encore véritablement une science, mais n'est plus tout à fait « humain ».

\section{CONCLUSION}

$\mathrm{Au}$ terme de ces considérations, je conclurai en disant que la traduction est parvenue au stade de maturité auquel les traducteurs ont tant aspiré : désormais ils assument leurs propres responsabilités, reconnaissent leurs limites et se tournent vers les éléments de solution qu'offrent d'autres disciplines. Ensuite, les efforts enfin convergents des praticiens et des théoriciens ont permis de fixer les limites du champ d'investigation des systèmes théoriques et ouvert l'éventail des possibilités de recherche en intégrant dans l'analyse de l'opération traduisante les données les plus récentes de la science ainsi que de disciplines nouvelles ou traditionnelles. De la sorte sont établis le statut quasi scientifique et le caractère spécifique de la traduction, une des rares disciplines qui puisse se réclamer à la fois de l'art et de la science et qui correspond ainsi à la fonction supérieure de la traduction qu'avait dégagée Heidegger, pour lequel

$/ . . . /$ une traduction ne consiste pas simplement à faciliter la communication, mais $/ . . . /$ est en soit un défrichement de la question posée en commun. Elle sert à la compréhension réciproque en un sens supérieur. Et chaque pas dans cette voie est une bénédiction pour les peuples. (Martin Heidegger, l'Être et le temps.)

Notes

1. Paul A. Horguelin (1981) : Anthologie de la manière de traduire. Domaine français, Montréal, Linguatech, p. 17) fait état de lexiques bilingues et même trilingues découverts sur le site de l'ancienne cité d'Ebla, dans le nord de la Syrie, datant du troisième millénaire avant Jésus-Christ. Voir aussi Time, 21 sept. 1981 , p. 82.

2. Voir sur cette question la communication présentée lors du Ve congrès mondial de la Fédération internationale des professeurs de français (Rio de Janeiro, 19-24 juillet 1981) : "Qualité linguistique et traduction - Fonctions de la traduction en milieu bilingue : le cas du Québec. » $\bar{A}$ paraitre dans les actes du congrès. 
3. Michel Foucault (1966) : les Mots et les choses, Paris, Gallimard, NRF, coll. " Bibliothèque des sciences humaines ", p. 355 et suiv.

4. Pour le domaine français, voir note 1.

5. Anthologie de la manière de traduire, op. cit., p. 19.

6. Ibid., p. 23.

7. Ibid., p. 23.

8. J.-P. Vinay dans un article publié dans Meta (" Regards sur l'évolution des théories de la traduction depuis vingt ans ", Meta, vol. 20, no 1, 1975, pp. 7-27) appelle ainsi les linguistes de profession qui cherchent à défricher le domaine de la traduction. Cet article est complété par une importante bibliographie où l'auteur recense les ouvrages, surtout théoriques, majeurs du domaine traductionnel.

9. Ibid., p. 8 .

10. A.V. Fedorov (1953): Vvedenie v torju perevoda [Introduction à la théorie de la traduction], Moscou; J.-P. Vinay et J. Darbelnet (1958) : Stylistique comparée du français et de l'anglais, Paris, Didier ; E. A. Nida (1964) : Toward a Science of Translating, Leiden, E.J. Brill.

11. Sur cette question voir plus particulièrement Georges Mounin (1963) : les Problèmes théoriques de la traduction, Paris, Gallimard, 290 p., rééd. 1976, coll. TEL, p. 21.

12. Ibid., p. 8 .

12. Charles P. Bouton (1979) : la Signification, Paris, Klincksieck, p. 26.

14. Barbara Ward et René Dubos (1971) : Nous n'avons qu'une terre [traduction de Only One Earth], Paris, Denoël.

15. Georges Mounin, op. cit., p. 169.

16. Louis G. Kelly (1979) : The True Interpreter. A History of Translation Theory and Practice in the West, Oxford, Basil Blackwell.

17. Op. cit. et avec Charles R. Taber (1969) : The Theory and Practice of Translation, Leiden, Brill, $220 \mathrm{p}$. (Traduction française : la Traduction: théorie et méthode, Londres, Alliance biblique universelle, 1971.)

18. Danica Seleskovitch (1978) : « Pour une théorie de la traduction inspirée de sa pratique », Meta, vol. 25, no 4, pp. 401-408; Maurice Pergnier (1978) : les Fondements sociolinguistiques de la traduction, Paris, Champion, 491 p.; Jean-René Ladmiral (1979) : Traduire : théorèmes pour la traduction, Paris, Petite bibliothèque Payot, $\mathrm{n}^{\circ} 366$; Jean Delisle (1980) : l'Analyse du discours comme méthode de traduction, Ottawa, Éditions de l'Université d'Ottawa, coll. "Cahiers de traductologie".

19. Voir plus particulièrement sur cette question le numéro spécial de Meta: l'Informatique au service de la traduction. Machine Aids to Translation, vol. 26, n 1 , mars 1981 